\title{
Ten Rules Preventing COVID-19
}

\author{
Iraj Salehi-Abari ${ }^{*}$, Shabnam Khazaeli ${ }^{2}$ \\ ${ }^{1}$ Rheumatology Research Center, Faculty of Medicine, Tehran University of Medical Sciences, Tehran, Iran \\ ${ }^{2}$ General Practitioner, Genetic Consultant, Toronto, Canada \\ Email: ^salehiabari@sina.tums.ac.ir, shabnam.khdoc@yahoo.com
}

How to cite this paper: Salehi-Abari, I. and Khazaeli, S. (2020) Ten Rules Preventing COVID-19. Advances in Infectious Diseases, 10, 123-128. https://doi.org/10.4236/aid.2020.103012

Received: July 10, 2020

Accepted: August 9, 2020

Published: August 12, 2020

Copyright $\odot 2020$ by author(s) and Scientific Research Publishing Inc. This work is licensed under the Creative Commons Attribution International License (CC BY 4.0).

http://creativecommons.org/licenses/by/4.0/ (c) (i) Open Access

\begin{abstract}
The new Coronavirus disease or COVID-19 is a contagious viral/immunological systemic disorder with predominantly respiratory features caused by human infection with SARS-CoV-2, which is rapidly spreading from person-to-person all around the world as a pandemic. As long as there is no effective vaccine available to prevent this disease and no definitive treatment is provided to cure it, it is better to prevent this disease by following a series of principles, which are: home quarantine of asymptomatic to mild COVID-19 patients and hospitalization of severe cases, physical distancing, staying at home, handwashing, cleaning and disinfecting high-touch surfaces, face-mask using, cough and sneeze covering, healthy nutritious diet along with the routine life activities and daily exercise, video communication by phone/online with family, and finally practice to relieve stress/anxiety.
\end{abstract}

\section{Keywords}

Quarantine, Telemedicine, Physical Distancing, Prevention, COVID-19, Face-Mask, Handwashing, High-Touch Surfaces

\section{Introduction}

The new Coronavirus disease is a contagious viral/immunological systemic disorder with predominantly respiratory features caused by human infection with a novel and emerging virus from Beta RNA-Coronavirus group; SARS-CoV-2, that is rapidly spreading from person-to-person all around the world as a pandemic. The SARS-CoV-2 is transmitted from human to human in various ways:

1) Airway: After coughing and sneezing, the viruses are thrown out with macro droplets of saliva (5 - 10 micrometers) and landing on the ground immediately up to a distance of 1 meter.

2) Aerosol way or airborne transmission: while talking and breathing (exhaled phase) the viruses are released up to 4 meters in the air with tiny micro-droplets 
of saliva ( $<5$ micrometers) and they can remain viable/float for up to 3 hours. It should be noted that micro-droplets of saliva can be thrown into the air up to 8 meters after sneezing.

3) Touching way: close personal contact such as shaking hands, touching, and kissing. Touching objects or surfaces contaminated with the virus by hands and then, touching infected hands with the mouth, nose, or eyes before washing hands.

4) Fecal-oral: The virus exits through the anus and into the stool of the person carrying it. Contamination of surfaces, hands, or food with feces containing the virus will transmit the virus. It is even claimed that the intestinal gas passing from the anus is another way of transmission [1] [2] [3].

5) Mother to child: This vertical transmission can occur during childbirth or breastfeeding.

6) Hospital-acquired: The virus can be transmitted to hospitalized patients, medical staff, and all other hospital staff, patient companions, and other clients in the hospital.

7) Community-acquired: If based on history, there is no acceptable evidence to justify how the virus is transmitted; it is referred to as community-acquired.

As long as there is no effective vaccine available to prevent this disease and no definitive treatment is provided to cure it, it is better to prevent this disease by following a series of principles.

The Persian scholar of medicine Ebne Sina (980-1037) suspected that some diseases were spread by microorganisms, and to prevent human to human contamination he came up with the method of isolating people for 40 days in Persian is called Cheleh. Traders from Venice saw his success and took this knowledge back to Italy and called it quarantine (forty in Italian). This is the origin of the word quarantine to fight pandemics, another bright idea coming from ancient Persia (Iran). Also, the historical history of using face masks goes back to the civilization of ancient Iran [4].

\section{Methods}

The principles of prevention of becoming infected with the SARS-CoV2 as Ten Rules Preventing COVID-19 include:

- Home quarantine of asymptomatic to mild COVID-19 patients and hospitalization of severe cases: Self-isolation of asymptomatic to mild COVID-19 patient from others in a specific room as sick room, with face-mask and with no visitors, and if available, with a separate bathroom, while his/her communication with the first level of COVID-19 Referral System or Family Physician could be continued by phone (telemedicine). Resting, warm-keeping, humidifier using and hot shower taking for cough and sore throat, hands cleaning after nose blowing or coughing or sneezing or use the bathroom and especially before eating or preparing foods, drinking plenty of fluids, a healthy nutritious diet and if needed symptomatic therapy are the only treatments available during this period. It is necessary to explain that home quarantine 
for parents with COVID-19 will not be effective without children's quarantine in another room at home [4] [5] [6] [7].

- Physical (social) distancing:

- Being away from others: $>2$ meters (acceptable) and, if possible, $>4$ meters (ideally)

- Do not gather in groups

- Prohibition of entering crowded places

- Public transportation and taxis as ride-sharing cannot be used at all [4] [5] [6] [7].

- Staying at home, especially for the elderly and children ages 15 to 20 years old or less than it, of course with the prohibition of coexistence of the elderly and children in a place under one roof, and also by establishing contactless meetings among residents [4] [5] [6] [7].

- Handwashing with water and soap for at least 20 seconds, and if they are not available, hand sanitizing with spray, gel or solutions containing at least $60 \%$ alcohol, especially before eating or preparing food and after nose blowing or coughing or sneezing or use the bathroom, and prohibition of face touching especially mouth, nose, and eyes with unwashed hands [4] [5] [6] [7].

- Cleaning and disinfecting high-touch surfaces every day including; door-knobs, handles, tables, light switches, countertops, desk, phone, keyboards, toilets, faucets, and sinks [4] [5] [6] [7].

- Face-Mask using:

- Medical (Surgical) masks for the general population when forcing to leave the home and in the workplace and community.

- N95 masks for health care professionals.

- Hands should be washed or disinfected before and after applying the mask to the face, as well as before and after removing the mask [4] [5] [6] [7].

- Cough and sneeze covering with a tissue or inside of your elbow, whenever you don't have a mask on your face [4] [5] [6] [7].

- Healthy nutritious diet along with the routine life activities and daily exercise [4] [5] [6] [7].

- Have a video communication by phone/online with family and friends and regular daily schedule to watch movies [4] [5] [6] [7].

- Practice to relieve stress/anxiety in these ways:

- Think positive.

- Accept the existing reality.

- Follow novel Corona news, only once or twice a day.

- Deep breathing.

- Doing yoga/muscle relaxation.

- Meditation.

- Call your doctor or your best friend to ask for help.

- Conversation with God, alone and beyond the window of the room, look- 
ing at the sky or the mountain [4] [5] [6] [7].

\section{Results}

Following a contact; the transmission probability of virus:

1) From a SARS-CoV-2 carrier without a mask, to a healthy person with a mask is $70 \%$

2) From a SARS-CoV-2 carrier with a mask, to a healthy person without a mask is $5 \%$

3) From a SARS-CoV-2 carrier with a mask, to a healthy person with a mask is $1.5 \%$

4) From a SARS-CoV-2 carrier without a mask, to a healthy person without a mask is $\geq 90 \%$ [3] [4] [5] [6] [7].

In the cases with positive SARS-CoV-2 kit test but without any symptoms/signs or with mild clinical features, four options could be possible:

1) Carrier state for SARS-CoV-2.

2) Subclinical COVID-19.

3) Pre-symptomatic COVID-19 within the incubation period.

4) Mild COVID-19.

The above cases are silent spreaders, who have a major role in the spread of SARS-CoV-2. In other words, they can spread SARS-CoV-2 for up to 2 weeks [3] [4] [5] [6] [7]. If such a person passes by an average of 100 people daily without physical distancing, and both face-masks are not worn; there is a $90 \%$ chance, and if only the silent spreader is without a face-mask; there is a 70\% chance that the other person will get it. This means that 90 and 70 people will be infected with the SARS-CoV-2 on a daily basis, respectively, of which; $40 \%$ will be asymptomatic, $40 \%$ will suffer from mild COVID-19, and $20 \%$ of cases will be severe/critical and cause hospitalization, and in the end, $5 \%$ of all these people will die. Then, if we multiply the resulting numbers by 14 , the results are two rows of numbers, which in order from left to right, indicate: the new cases of COVID-19 in the forms of; asymptomatic, mild, hospitalized candidates, and dead cases within 14 days:

$504, \quad 504, \quad 252, \quad 63$

$392, \quad 392, \quad 196, \quad 49$

This is due to the presence of an asymptomatic person infected with SARSCoV-2 that has the personalities of ignorant, thoughtless, selfish, incomprehensible, unconscious, or a complex person that is called "More Dangerous than a Zombie" who refuses to the face-mask is not applied. Of course, provided that the government financially supports the poor and needy in society.

Recovery (Discharge) Criteria for COVID-19: Patients with COVID-19 recover when:

1) Afebrile for at least the last 72 hours, without using fever-reducing medications and;

2) Respiratory symptoms, especially coughing and shortness of breath, im- 
proved and;

3) More than one week has passed since the onset of symptoms;

4) Significant improvement of acute lesions on chest HRCT;

5) Two consecutive negative RT-PCR tests at least 24 hours apart [3] [4] [5] [6] [7].

The fate of the COVID-19 patients after home quarantine or self-isolation at home is as follows:

1) Asymptomatic patients can terminate home quarantine after 2 weeks because they carry the virus for up to 2 weeks.

2) Mild patients can terminate home quarantine after 3 weeks because they carry the virus for up to 3 weeks. Provided, of course, that there are criteria for recovery.

3) Hospitalization of mild to moderate cases of COVID-19 who do not or cannot afford home quarantine, in semi-specialized or field Hospital of the level (II) of COVID-19 Referral System.

4) With the occurrence of initial alarming/warning symptom, on the other hand, shortness of breath (new or worsening, for no other cause); the patient must be hospitalized in Corona Hospital Centre of the level (III) of COVID-19 Referral system.

5) Severe to very severe patients should be quarantined in the Corona hospital and then at home for a total of at least 4 to 6 weeks. Provided, of course, that there are criteria for recovery.

So, asymptomatic and mild COVID-19 patients, who make up $80 \%$ of cases, recover after 2 to 3 weeks. Moderate to severe COVID-19 patients usually recover within 3 to 6 weeks but the recovery in critical cases of COVID-19 varies with the person [3] [4] [5] [6] [7].

\section{Conclusion}

According to the corresponding author, the combination of quarantine, staying at home, physical distancing, handwashing, face-mask using and cough/sneeze covering are the main foundation of COVID-19 prevention.

\section{Conflicts of Interest}

The authors declare no conflicts of interest regarding the publication of this paper.

\section{References}

[1] Gu, J.Y., Han, B. and Wang, J. (2020) COVID-19: Gastrointestinal Manifestations and Potential Fecal-Oral Transmission. Gastroenterology, 158, 1518-1519. https://doi.org/10.1053/j.gastro.2020.02.054

[2] Cicuttin, E., Cobianchi, L., Chiarugi, M., Catena, F., Coccolini, F. and Pietrabissa, A. (2020) Detect to Protect: Pneumoperitoneum Gas Samples for SARS CoV 2 and Biohazard Testing. Surgical Endoscopy, 34, 2863-2865.

https://doi.org/10.1007/s00464-020-07611-7 
[3] World Health Organization (2020) Transmission of SARS-CoV-2: Implications for Infection Prevention Precautions: Scientific Brief, 9 July 2020. No. WHO/2019nCoV/Sci_Brief/Transmission_modes/2020.3.

[4] Salehi-Abari, I., Khazaeli, S., Salehi-Abari, F. and Salehi-Abari, A. (2020) Practical Guideline for Screening the Patients with SARS-CoV-2 Infection and Persian Gulf Criteria for Diagnosis of COVID-19. Advances in Infectious Diseases, 10, 67-93. https://doi.org/10.4236/aid.2020.103008

[5] Coronavirus: Information \& Resources, National Jewish Health. COVID-19 (Coronavirus) Antibody \& Molecular Diagnostic Testing Available.

[6] CDC: Centers for Disease Control and Prevention CDC 24/7: Saving Lives, Protecting People; Coronavirus COVID-19.

[7] WHO (2020) News and Events regarding COVID-19 and SARS CoV-2. 